\title{
Mass-Measurement-based Automatization of the Engler-Viscometer
}

\author{
Katalin Harangus, András Kakucs
}

Sapientia Hungarian University of Transylvania, Faculty of Technical and Human Sciences, Op. 9, Cp. 4, 540485 Târgu-Mureș, Romania

e-mail: katalin@ms.sapientia.ro, kakucs2@ms.sapientia.ro

\begin{abstract}
The Engler-viscometer, as one of the efflux-type viscometers, in general, was introduced and standardized, more than 100 years ago. The principle of the measurements is based on the efflux time, of a certain volume of liquid trough a short capillary tube, placed on the bottom of the cup of the viscometer. The viscosity of the liquid is given in Engler-degrees as the ratio of the efflux time of the measured liquid and that of the distillated water at $20{ }^{\circ} \mathrm{C}$. There is no direct proportionality between the efflux time and viscosity because the Reynolds-number is not the same. This usually is transformed in "true" viscosity units using some semi-empirical formulas. The technical literature of these types of viscometers, especially of the Engler-viscometer, is very poor, but in these papers and books, certain doubts are often mentioned, concerning the accuracy of these formulas. One of the sources of the error is volume measurement, especially for the less-viscous liquids, for example, water. We have proposed a new, mass-measurement based approach that does not need the modification of the viscometer and it also eliminates the negative effect of the thermal expansion of the liquid. This makes possible, a fully automatized measurement and because it provides the evolution in time, of the collected quantity, it also helps in the deeper investigation of the efflux time - viscosity relation.
\end{abstract}

Keywords: Engler-viscometer; modernization; mass-measurement

\section{Efflux-Type Viscometers}

The Engler viscometer is a "capillary" type, categorized as "efflux type", mainly used in determination of the viscosity of petroleum products. Based on some earlier viscometers, it was developed by the German chemist, Carl Oswald Victor Engler (1842-1925) around 1890, following his studies in petrochemistry, in 1884. He published the description of this apparatus in "Normen für das Viscosimeter" (Standards of the viscometer) in "Zeitschrift für Angewandte Chemie" (Journal of Applied Chemistry) in 1892. This viscometer became the standardized one, widely used in the world, especially in the continental European Countries. 
A few years earlier, in 1885-1886, sir Thomas Boverton Redwood (1846-1919), focusing on some technical problems regarding petroleum products, had improved an older efflux type viscometer in use. This improved version became a standard laboratory apparatus in England and in many other countries. For very viscous fluids, this type of viscometer was modified leading to the "Redwood No. 2": The main differences entail the increase of the diameter of the evacuating tube (that of the "jet") and in the modification of the dimensions of the reservoir (those of the "cup") in order to reduce the pouring time of the target fluid.

In the United States of America, a third efflux-type apparatus was standardized, the Saybolt Universal viscometer that also has two versions with enlarged diameter of the jet for more viscous fluids, the Saybolt Furol and Saybolt Asphalt viscometers.

All of these viscometers work, based on the same principle: The efflux time of a certain standardized volume of liquid, is directly related to the viscosity of that liquid, so the viscosity is computed or appreciated, based on the measured efflux time.

They work in a similar manner, only some of the construction details are different [4].

They consist of a cup (vertical cylindrical reservoir, denoted " $A$ " in case of the Engler viscometer in figure 1) with standardized sizes, opened on the top and closed by a standardized shape bottom, covered by a thermo-insulated lid $(B)$. The cup of the early Engler and Redwood viscometers was plated inside with gold or silver, nowadays they are made of cheaper corrosion-resistant stainless steel. The cup of the Saybolt viscometers is made of brass or other corrosion-resistant metal. This cup is placed in a thermostatic chamber filled with water or oil $(C)$ and provided with a thermometer $(D)$ and a stirrer $(E)$ to obtain a uniform temperature in the thermostatic bath. The fluid usually is heated electrically, in the case of some versions, it can also be cooled.

The internal diameter of the Engler viscometer cup is $a=106 \mathrm{~mm}$ and the height of its cylindrical part is $b=25 \mathrm{~mm}$. The volume of this cylindrical part is $220.5 \mathrm{~cm}^{3}$ that is $10 \%$ larger than the volume to be collected during the measurements (this standardized volume is $200 \mathrm{~cm}^{3}$ ).

On the bottom of the cup there is a central hole where a short vertical capillary (the jet, $F$ ) is fitted. This also has some standardized dimensions and it is made of non-corrosive material. The jet of the early Engler viscometers was made of platinum or brass covered inside with thick platinum coating. Nominally, its length is $20 \mathrm{~mm}$ and its internal diameter is $2.8 \mathrm{~mm}$ at the lower end. On the upper end its diameter is $0.1 \mathrm{~mm}$ larger $(2.9 \mathrm{~mm})$. Its external diameter is $4.5 \mathrm{~mm}$. The lower end is $c=52 \mathrm{~mm}$ under the maximum level in the cup and $d=3 \mathrm{~mm}$ under the bottom of the thermostatic bath [3]. 
The traditional material of the jet of the Redwood viscometer is agate. The Saybolt viscometers are made entirely of corrosion-resistant metal.

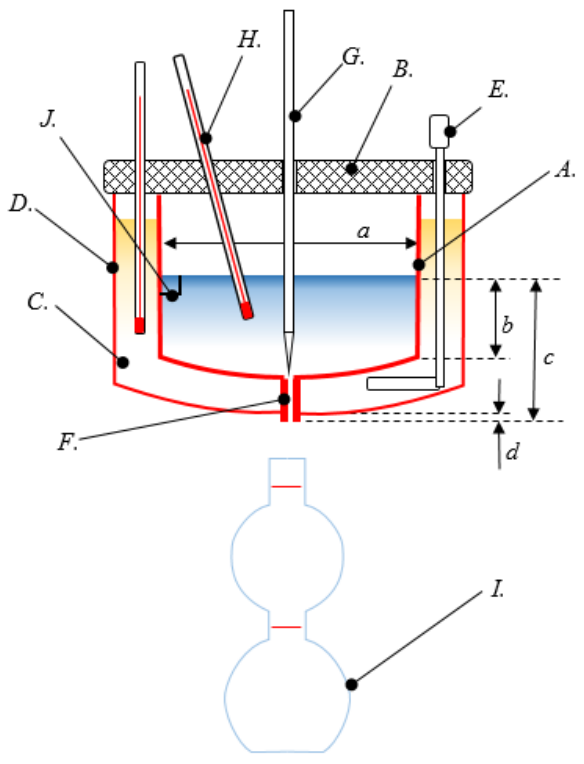

Figure 1

Schematics of the Engler viscometer

The jet in the case of the Engler viscometer is closed in its upper end, inside the cup, by a valve rod $(G)$ that can be vertically lifted through the lid.

The jet of the Redwood viscometer is controlled by a small metallic ball resting in a hemispherical cavity of the agate, on its superior end. This can be lifted using the attached wire.

So given, at the beginning of the measurements, the capillary tube in case of these viscometers is empty.

The Saybolt viscometer has a cork stopper placed under the jet that can be pulled out downward using a small chain or a rope. This stopper has to be fitted as well as it prevents escaping the air; this is evidenced by the absence of the measured liquid on the stopper when it is removed at the beginning of the measurement but it does not guarantee that the jet is empty.

All viscometers are provided with a thermometer placed inside the cup $(H)$, a three-legged stand with leveling screws (not shown in Figure 1) and a measuring flask $(I)$.

The cup has to be filled with a certain volume of liquid. In the case of the Engler viscometer the corresponding level is indicated by three pointers $(J)$ used also in leveling, while the Redwood viscometer has only a single gauge. This pointer is a 
piece of sharpened nail (wire) bent upwards, fixed on the inner surface of the cup. The volume in the Saybolt viscometer is controlled by an overflow-rim.

The measuring flask of the Engler viscometer is a two-bulb glassware with a short narrow neck between the bulbs and a longer one at its mouth. Between the two bulbs there is a mark corresponding to $100 \mathrm{~cm}^{3}$ of volume. Above the upper bulb on the neck there is another mark indicating the $200 \mathrm{~cm}^{3}$ of the collected liquid poured out from the cup.

The flask of the Redwood viscometer is a $50 \mathrm{~cm}^{3}$ volumetric one. The Saybolt viscometer has a flask of $60 \mathrm{~cm}^{3}$ capacity that has a funnel-like mouth.

The standard testing method begins with preparing the sample (e.g. by filtering if this is necessary), cleaning and drying the cup, the jet and the flask of the apparatus. The thermostatic bath must be set up by filling with oil or water. The volumetric flask has to be placed beneath the jet. The cup has to be filled to the level indicated by the pointer(s) or set by the overflow rim, while the jet is closed by the valve rod, the metallic ball or the cork, respectively. The thermostatic bath has to be heated or cooled applying some thermal control and stirring to reach and keep the desired temperature in an allowable range. When the temperature is set, the jet is opened and a chronometer is started in the same time. This chronometer is stopped when the level of the effluent liquid reaches the mark on the measuring flask.

In the case of the Engler viscometer, the viscosity is given as the ratio of the $t$ efflux time of the measured liquid and that $t_{0}$ of the distillated water at $20^{\circ} \mathrm{C}$. This is expressed in Engler degrees $\left({ }^{\circ} \mathrm{E}\right)$ and usually it is transformed in "true" viscosity units (e.g. poise or $\mathrm{m}^{2} / \mathrm{s}$ for cinematic viscosity) using some experimental tables, charts or relations like:

$v_{k}=A \cdot E-\frac{B}{E}, E=t / t_{0}$

Are obtained by calibration using liquids with known viscosity (Ubbelohde's formula).

In the case of Redwood viscometer, the efflux time is given in "Redwood seconds". The efflux time of the Saybolt viscometer is corrected with an experimental calibration factor obtained using some standard oils, obtaining the measure of the cinematic viscosity in "Saybolt X seconds" or "Saybolt X viscosity" units, where "X" stands for "Universal", "Furol" or "Asphalt" depending on the apparatus used. The "Redwood seconds" and "Saybolt seconds" are transformed in kinematic viscosity by the formula of above as:

$v_{k}=A \cdot t-\frac{B}{t}$ 


\section{Disadvantages of Efflux-Type Viscometers}

There are often enumerated, some technical issues, as disadvantages of these viscometers, since the difficulty of cleaning the capillary tube and so on, but studying how these are working, some other issues can be enumerated.

A century ago (in 1921), Winslow H. Herschel has written a short monography entitled "The Redwood viscometer", published by the U.S. Government Printing Office in 1922 (also republished by the Forgotten Books in 2019) [1], concerning the standardization of this type of viscometers. He paid attention to the "cooling error" that is the negative effect of the changes in temperature of the effluent liquid. The principle of these viscometers is based on the measuring the efflux time of a certain volume of fluid on certain set temperature, but the volume is measured using a collecting flask that is non thermostatic as the cup is. At the end of the measurement, the difference between the nominal temperature of the test and that of the liquid in the flask depends upon a series of factors. As the difference between the testing temperature increases and the viscosity is higher, the error will be higher due to the shrinking of the liquid and to the dilation of the collecting flask. This error in the mentioned monography is up to $5 \%$ in case of oils tested at $100^{\circ} \mathrm{C}$.

To reduce this error, some solutions were proposed, such as, using a double walled glassware as a collecting flask or providing it with a thick thermo-insulator cover.

Another disadvantage of these viscometers, is the reading error of the level in the collecting flask. The chronometer must be stopped when the effluent liquid reaches the mark that is appreciated by human eye reading. The impediments are the meniscus, the foam, the presence of air bubbles and those of the liquid drops on the wall in case of rapidly pouring liquids with reduced viscosity (this is the case of the water used in calibration of the Engler viscometer, Figure 2).
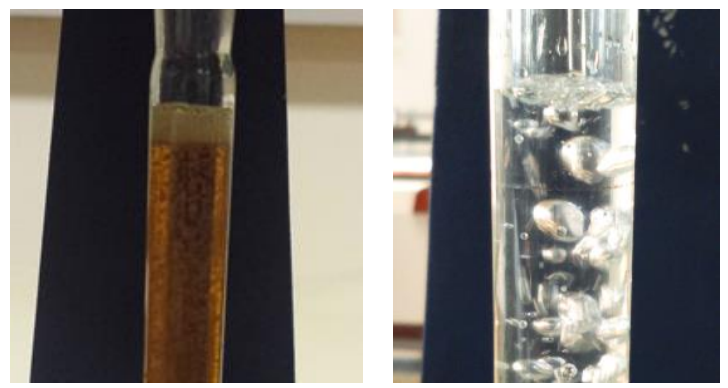

Figure 2

Bubbles and foam in the collecting flask 
The human reaction time in starting-stopping the chronometer is also a source of the reading errors: it is said that normally this is $0.7 \mathrm{~s}$ that is more than $1 \%$ of the cca. $50 \mathrm{~s}$ efflux time of the water in the calibration process of Engler viscometer.

The main disadvantage of efflux viscometers, is found in the difficulty of calibration. The concept of the hydraulic similitude cannot be applied in their calibration because the outflowing of two different liquids happens with different Reynold's numbers, so the viscosity cannot be computed using scale ratios.

\section{A New Method in Measuring the Efflux Time}

Because of the difficulties generated in the detection of the exact volume of the liquid collected in the flask, we have proposed a new method based on mass (weight) measurement, without any modification of the viscometer. This is done by using a scale placed under the collecting flask. This scale is built with a loadcell, having four strain-gauges, wired as a full Wheatstone-bridge. This is connected to a specialized load-cell amplifier, that has an analog-to-digital converter circuit (e.g. HX711). The digital output of this circuit is read and saved in a file by a data acquisition and controlling system built around a microcontroller (e.g. ATmega2560 or ESP32). To avoid the acquisition of useless data a micro-switch ought to be placed on the viscometer to detect the position of the valve rod: as the rod is lifted the data acquisition starts and this is stopped when the valve is pushed back into its closed position. Using the same microcontroller and some circuitry, there is also the possibility to resolve the controlling of the thermostatic bath.

In this way the out flown volume of the liquid can be computed as:

$V(t)=\frac{m}{\rho(t)}$

Where, $m$ is the acquired mass and $\rho(t)$ is the density of the liquid in the cup (at the set temperature $t$ ). When $t$ is not equal to the room temperature the volume collected in the flask is not equal to this computed value. In this way the problems regarding the reading of the level and the "cooling error" mentioned before are resolved but the $\rho(t)$ density must be known.

The viscosity is computed from the time $t$ corresponding to $V(t)=200 \mathrm{~cm}^{3}$. Because the mass is acquired with a certain rate, $V(\tau)$ is obtained by interpolation based on the registered data. 


\section{Pros and Cons of the New Method}

The main advantages of the proposed mass-measurement based volume detection are:

- It can be applied without any modification of the apparatus

- It can be automatized in a very simple way

- It eliminates the errors related to the thermal expansion of the liquid (the cooling error)

- It eliminates the level-reading errors, including the human reaction time, those produced by bubbles and by the foam

- There is no need of well-cleaned and dried calibrated collecting flask

- It makes possible to track the whole process as the liquid flows out from the cup (this may be useful in calibrating and theoretical investigations)

The main disadvantages are:

- The density of the liquid at the testing temperature must be known (measured)

- The acquired data is influenced by noise (because the collected and measured mass is continuously growing there is not possible to get a mean value from repeated samples as is usually done in case of digital balances)

- The efflux time of the standardized $200 \mathrm{~cm}^{3}$ is obtained by processing the acquired data

- The mass indicated by load cell is slightly biased by the momentum $p$ of the falling jet or that of the drops

- In case of less viscous liquids (as water at $20^{\circ} \mathrm{C}$ ), the lower part of the jet may be turbulent and this turbulence may cause some noise

- In case of higher viscosity, instead of a continuous jet the liquid falls in individual drops that can also generate some noise as the dynamic excitation of the low-damped scale mechanism

The negative influence of the phenomena mentioned in the last three disadvantages, may be reduced, by using a flat dish-like collecting vessel, as a flat beaker or crystallizer, placed close under the bottom of the viscometer. 


\section{Efflux Time vs. Viscosity}

The process of outflowing is not a stationary one because of the decreasing head as the liquid leaves the cup during the measurements, so the exact analytical computation is not possible because we need to know the streamlines at each moment of the computation. So the first approximation of this computation can be done by using the Bernoulli's principle in its simplest form for stationary flows:

$$
\frac{\alpha_{1} \cdot v_{1}^{2}}{2 \cdot g}+\frac{p_{1}}{\gamma}+z_{1}=\frac{\alpha_{2} \cdot v_{2}^{2}}{2 \cdot g}+\frac{p_{2}}{\gamma}+z_{2}+h_{r 1-2}
$$

In this equation:

$-\alpha_{i}$ are the Coriolis coefficients adjusting the kinetic energy computed from the mean velocity to that computed by taking the true velocity distribution on the cross section of the flow. In laminar flow in circular tubes the value of this coefficient is computed based on the Hagen-Poiseuille velocity distribution and it is $\alpha=2$

$-v_{i}$ are the mean velocities on the two cross sections. If we take the first one on the free surface of the liquid in the cup and the second one on the lower end of the evacuating capillary tube, the ratio of cross sections with $d_{1}=106 \mathrm{~mm}$ of the cup and $d_{2}=2.8 \mathrm{~mm}$ as the diameter of the lower end of the capillary is:

$$
\frac{A_{1}}{A_{2}}=\left(\frac{106}{2.8}\right)^{2} \approx 1433
$$

and the $v_{1}$ velocity is less than $0.1 \%$ of $v_{2}$. So given, the specific kinetic energy on the first cross section is less than one millionth part of that at outflowing and it can be neglected by taking $v_{1}=0$. Let denote the $v_{2}$ mean velocity as " $v$ ", computed with the volumetric flow rate and the cross section of the capillary as:

$v=4 \cdot Q_{V} /\left(\pi \cdot d^{2}\right)$

- $p_{i}$ are the hydrostatic pressure that is the atmospheric one on both sections;

$-h=z_{1}-z_{2}$ is the hydraulic head as the vertical distance from the free surface in the cup to the lower end of the tube. This varies from $52 \mathrm{~mm}$ at the beginning of the measurements to $29.3 \mathrm{~mm}$ at the end when the $200 \mathrm{~cm}^{3}$ of the liquid is collected in the flask;

$-h_{r 1-2}$ is the head loss. Because inside the cup the velocity is very low and the cross section is relatively large, this loss is produced at the evacuating capillary tube. Using the Darcy-Weissbach equation this is computed related to the kinetic energy as: 
$h_{r}=\zeta \cdot \frac{v^{2}}{2 \cdot g}$

where:

$\zeta=\zeta_{t}+\zeta_{l}$

is the sum of the flow coefficient of the tubes and those locally.

In our case there is only one local source of the energy losses that is the contraction at the entrance in the capillary, which has a flow coefficient of: $\zeta_{l}=0.5$

The flow coefficient for circular tube with the length $l$ and diameter $d$ is expressed using the $\lambda$ friction factor as:

$\zeta_{t}=\lambda \cdot \frac{l}{d}$

Based on the Hagen-Poiseuille velocity distribution the friction factor in laminar flow for circular tubes is:

$\lambda=\frac{64}{\operatorname{Re}}$

where the expression of the Reynold's number is:

$\operatorname{Re}=\frac{v \cdot d}{v_{k}}$

By taking an average diameter $d=2.85 \mathrm{~mm}$ of the capillary and $\mathrm{Re}=2000$ (this is the approximate value of the Reynold's number at the beginning of testing with water), with its length of $l=20 \mathrm{~mm}$ the flow coefficient of the evacuating tube is

$\zeta_{t}=\frac{64}{\operatorname{Re}} \cdot \frac{l}{d}=0.228$

that is less than that for the contraction at the entrance. For higher viscosity oils the Reynold's number is greater and the head loss of the capillary became predominant over the local loss of above.

With all these relations we obtain the formula of the head loss with:

$h_{r}=\frac{32 \cdot l}{g \cdot d^{2}} \cdot v_{k} \cdot v+\frac{\zeta_{l}}{2 \cdot g} \cdot v^{2}$

and the Bernoulli's equation takes the form: 
$h=\frac{\left(\alpha+\zeta_{l}\right) \cdot v^{2}}{2 \cdot g}+\frac{32 \cdot l}{g \cdot d^{2}} \cdot v \cdot v_{k}$

or, with the flow rate $Q_{V}$

$h=\frac{8 \cdot\left(\alpha+\zeta_{l}\right) \cdot Q_{V}^{2}}{\pi^{2} \cdot d^{4} \cdot g}+\frac{128 \cdot l}{\pi \cdot g \cdot d^{4}} \cdot Q_{V} \cdot v_{k}$

So the kinematic viscosity can be expressed as:

$v_{k}=\frac{\pi \cdot g \cdot d^{4} \cdot h}{128 \cdot l} \cdot \frac{1}{Q_{V}}-\frac{\alpha+\zeta_{l}}{16 \cdot \pi \cdot l} \cdot Q_{V}=A \cdot \frac{1}{Q_{V}}-B \cdot Q_{V}$

that can be regarded as a detailed form of the Ubbelohde's formula (1).

The main problem with this formula is that nothing guarantees that the flow in the capillary tube follows the Hagen-Poiseuille velocity distribution. It is deduced using the hypothesis of the steady-state flow by neglecting the

$\int_{1}^{2} \frac{\partial v}{\partial t} d s$

quantity (because of the variable cross section and of the decreasing level in the cup, this integral cannot be computed analytically). This formula gives the value of the kinematic viscosity at a certain moment $\tau$ for the actual values of $h=h(\tau)$ and $Q_{V}=Q_{V}(\tau)$. Following the standardized process of the measurements these values are unknown, only the efflux time $t$ of the volume $V$ is determined. With this time an average value of the volumetric rate flow can be computed as:

$\bar{Q}_{V}=\frac{V}{t}$

and some attempts were made to establish a mean value $\bar{h}$ of the head. For the Redwood viscometer by using these mean values and a corrected value $\bar{l}$ of the length of the tube it was proposed the

$v_{k}=\frac{\pi \cdot g \cdot d^{4} \cdot \bar{h}}{128 \cdot \bar{l}} \cdot \frac{1}{\bar{Q}_{V}}-\frac{m}{8 \cdot \pi \cdot \bar{l}} \cdot \bar{Q}_{V}=A \cdot \frac{1}{Q_{V}}-B \cdot Q_{V}$

formula [1], where $B$ is corresponding to $\alpha+\zeta_{l}=2 \cdot m$ by referencing the $\left(17^{\text {th }}\right)$ formula. Here $m$ is a correction coefficient of the kinetic energy. The corrected value $\bar{l}$ is an increased one of the length of the tube that includes the effect of the contraction at the entrance in the capillary. Some details are given in [2] too. In the case of the Engler viscometer the variable diameter of the tube also has to be taken with an average $\bar{d}$ value. 
With the (18) average rate flow this can be transformed into a relation that gives the kinematic viscosity as the function of the efflux time:

$v_{k}=\frac{\pi \cdot g \cdot d^{4} \cdot \bar{h}}{128 \cdot \bar{l} \cdot V} \cdot t-\frac{m \cdot V}{8 \cdot \pi \cdot \bar{l}} \cdot \frac{1}{t}=A \cdot t-\frac{B}{t}$

Accepting the (14) formula the mean velocity on the cross section of the capillary tube can be computed using a step-by step approximation:

$v\left(t_{i+1}\right)=\frac{-c_{1}+\sqrt{c_{1}^{2}+2 \cdot c_{2} \cdot h\left(t_{i+1}\right)}}{c_{2}}$

where:

$c_{1}=\frac{32 \cdot l \cdot v_{k}}{g \cdot d^{2}}$

$c_{2}=\frac{m \cdot\left(\alpha+\zeta_{l}\right)}{g}$

$h\left(t_{i+1}\right)=h\left(t_{i}\right)-\frac{V\left(t_{i}\right)}{A}$

When we use $l=20 \mathrm{~mm}$ (the real length of the capillary), $d=2.85 \mathrm{~mm}$ (averaged diameter), $A=8.824734 \cdot 10^{-3} \mathrm{~m}^{2}$ (the cross section of the cup calculated using its diameter $D=0.106 \mathrm{~m}$ ), $h(t=0)=0.052 \mathrm{~m}$ (the head of the filled viscometer), $v_{k}=1.0034 \cdot 10^{-6} \mathrm{~m}^{2} / \mathrm{s}$ (water at $20^{\circ} \mathrm{C}$ ), $\alpha=2$ (HagenPoiseuille), $\zeta_{l}=0.5$ (contraction) and $m=1$ (as correction factor) the efflux time results $t=59.3 \mathrm{~s}$, much more than the expected $52 \mathrm{~s}$. With the value of $m=0.75$ of the correction factor the computed efflux time is $51.8 \mathrm{~s}$, closer to the desired one, leading to the

$A=1.072 \cdot 10^{-5} \mathrm{~m}^{2} / \mathrm{s}, \quad B=7.187 \cdot 10^{-6} \mathrm{~m}^{2} / \mathrm{s}$

coefficients of the (1) formula.

The coefficients published by Ubbelohde are quite different [2] [3]:

$v_{k}=0.073 \cdot E-\frac{0.0631}{E}[$ centistokes $]=0.73 \cdot 10^{-5} \cdot E-\frac{6.31 \cdot 10^{-6}}{E}\left[\mathrm{~m}^{2} / \mathrm{s}\right]$

referred in [2] as "theoretically derived" ones. If we introduce a correction factor of the coefficient $A$ (originally it was applied to get a "corrected length" of the capillary tube) there is a hope to get some results closer to the Ubbelohde's formula but these won't be purely theoretical ones. To investigate the role of the coefficients we rewrite eq. (16) as: 
$v_{k}=A^{\prime} \cdot h \cdot \frac{1}{Q_{V}}-B \cdot Q_{V}$

with,

$$
A^{\prime}=\frac{\pi \cdot g \cdot d^{4}}{128 \cdot l}=\frac{A}{h}
$$

Accepting that $A^{\prime}$ and $B$ are time-independent constants, for any $Q_{V, 1}=Q_{V}\left(t_{1}\right)$ and $Q_{V, 2}=Q_{V}\left(t_{2}\right)$ corresponding to the $t_{1} \neq t_{2}$ instants we must have,

$v_{k}=A^{\prime} \cdot h_{1} \cdot \frac{1}{Q_{V, 1}}-B \cdot Q_{V, 1}=A^{\prime} \cdot h_{2} \cdot \frac{1}{Q_{V, 2}}-B \cdot Q_{V, 2}$

leading to:

$\frac{A^{\prime}}{B}=\frac{Q_{V, 1} \cdot Q_{V, 2} \cdot\left(Q_{V, 1}-Q_{V, 2}\right)}{Q_{V, 2} \cdot h_{1}-Q_{V, 1} \cdot h_{2}}=$ const.

Using some acquired data we can observe that this ratio is not constant (Figure 3, drawn using smoothed experimental data for water at $20^{\circ} \mathrm{C}$, where $t_{2}=t_{1}+0.1 \mathrm{~s} \mathrm{)}$, demonstrating the fact that the steady-state version of the Bernoulli's equation cannot be used in exact modelling of the relation between the kinematic viscosity and the efflux time, so Ubbelohde's relation may not fit the actual data. This fact was experimentally observed [5] and a new formula was proposed:

$v_{k}=\frac{E}{100} \cdot 7.6^{1-1 / E^{3}} \quad$ [centistokes $]$

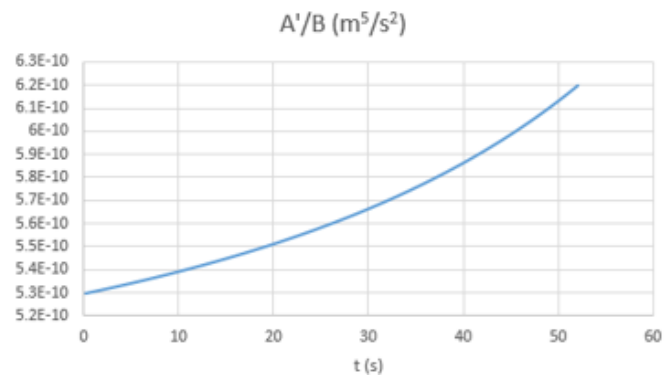

Figure 3

Evolution of the $\mathrm{A}^{\prime} / \mathrm{B}$ ratio

\section{Conclusions}

Experimental data was obtained for water, 10W40 motor oil and T90 transmission (gear) oil, all tested at room temperature $\left(20^{\circ} \mathrm{C}\right)$. The efflux time was measured using both the "classic" volumetric method and that based on mass measurement. 
As primary conclusions we can state the following:

- In the case of water, which has a higher outflow rate, even if there are some errors in the measured mass, the obtained results are closer to each other than those obtained by visual observation of the collected volume.

- In the case of higher viscosity oils the outflow rate is so small that changes can only be seen over a long period of time during the level reading.

- The noise of the electronic apparatus can be reduced by smoothing the acquired data.

The mean efflux time obtained for water was $50.6 \mathrm{~s}, 2034 \mathrm{~s}$ for motor oil, and $5051 \mathrm{~s}$ in the case of transmission oil. Those lead to a value of $40.2^{\circ} \mathrm{E}$ for the viscosity of the motor oil and that of $99.8^{\circ} \mathrm{E}$ for the transmission oil. For comparison, the standardized minimum of the kinematic viscosity of the tested motor oil at $20^{\circ} \mathrm{C}$ is $2.42 \cdot 10^{-4} \mathrm{~m}^{2} / \mathrm{s}$ (its maximum value is not specified by the standards, but the minimum for the $15 \mathrm{~W} 40$ motor oil is $2.87 \cdot 10^{-4} \mathrm{~m}^{2} / \mathrm{s}$ ). In the case of the transmission oil at this temperature, the range of the kinematic viscosity is given as $(5 \ldots 14) \cdot 10^{-4} \mathrm{~m}^{2} / \mathrm{s}$ (standardized values are specified only for higher temperatures).

Using Ubbelohde's (23) formula the kinematic viscosity is obtained as $2.93 \cdot 10^{-4} \mathrm{~m}^{2} / \mathrm{s}$ for the motor oil and $7.28 \cdot 10^{-4} \mathrm{~m}^{2} / \mathrm{s}$ for the transmission oil.

With (28), the obtained viscosities are slightly higher: $3.06 \cdot 10^{-4} \mathrm{~m}^{2} / \mathrm{s}$, respectively $7.58 \cdot 10^{-4} \mathrm{~m}^{2} / \mathrm{s}$.

The "nominal" density of the water on this temperature is $998.23 \mathrm{~kg} / \mathrm{m}^{3}$. The density of the oils was obtained by measurements: $875 \mathrm{~kg} / \mathrm{m}^{3}$ for the motor oil and $896 \mathrm{~kg} / \mathrm{m}^{3}$ for the transmission oil.

Figures 4 and 5 show the volumetric flow-rate obtained with the $m(t)$ collected mass as:

$\bar{Q}_{V}(t)=\frac{m(t) / \rho}{t}$

The curve obtained for the transmission oil is similar to that of the motor oil; (the outflow time of this oil was much longer).

Eq. (29) is a mean value at the time $t$ introduced based on the observation that for higher values $t$ the relative error in $m(t)$ is smaller. These graphs show an unsteady behavior in the initial phase that can be explained by the neglected component (17) of Bernoulli's equation and by the dynamic excitation of the scale mechanism. 
The observed noise of the used apparatus built around the HX711 load cell amplifier is approx. $0.03 \mathrm{~g}$ (as r.m.s. value), with peaks around $0.1 \mathrm{~g}$ (data obtained in steady conditions). The apparatus has the precision and the sensitivity of the technical scales, so it does not need special enclosure as an analytical balance does. Even in the case of a low-density liquid as the gasoline is $\left(\rho \approx 700 \mathrm{~g} / \mathrm{cm}^{3}\right)$, the relative error in the measured mass of the $200 \mathrm{~cm}^{3}$ liquid is under $1 \%$ (in steady conditions).

The theoretical value of the impact force of the jet can be computed as $F=\rho \cdot Q_{V} \cdot v$. One of the worst cases is that of the water at the beginning of the measurements because of the relatively high density and of the highest outflow velocity, when the jet hits the solid bottom of the collecting flask. In this case the estimated magnitude of the impact force is $F=1000 \mathrm{~kg} / \mathrm{m}^{3} \cdot 4.8 \cdot 10^{-6} \mathrm{~m}^{3} / \mathrm{s} \cdot 0.75 \mathrm{~m} / \mathrm{s}=0.0036 \mathrm{~N}$. This leads to a computed bias of approx. $0.37 \mathrm{~g}$ in mass measurement and also induces some vibrations of the load cell assembly. As the collected volume in the flask increases the outflow velocity becomes smaller and we can observe a continuously falling amplitude of the vibrations due to the increasing mass and of the viscous damping, and the effect of the falling jet or drops is buried into the general noise of the acquired data.

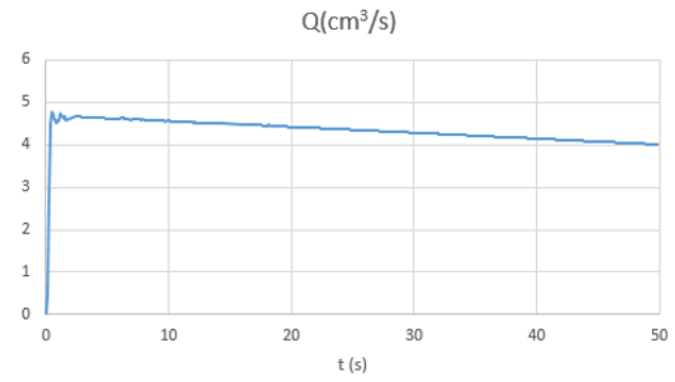

Figure 4

Mean flow-rate in case of $20^{\circ} \mathrm{C}$ water

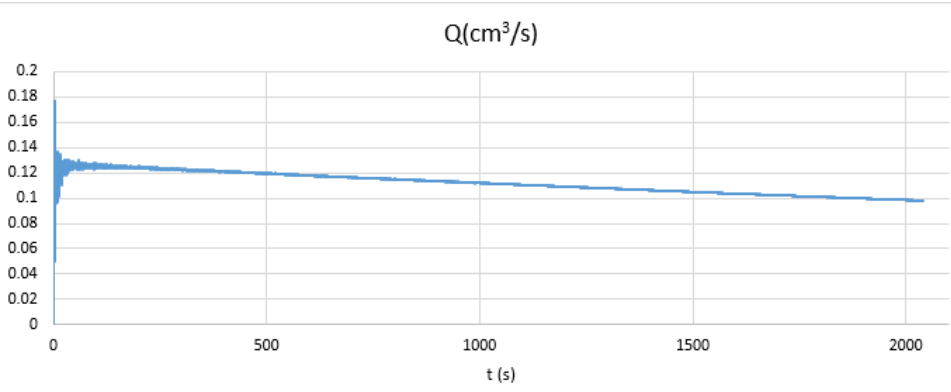

Figure 5

Mean flow-rate in case of a $10 \mathrm{~W} 40$ motor oil at $20^{\circ} \mathrm{C}$ 
To reduce these negative influences we focused on the outflow of the last $100 \mathrm{~cm}^{3}$ of liquid. It seems that the observed $V(t)=m(t) \cdot \rho$ volume is well approximated by a second order polynomial. The coefficients of this polynomial are smaller for higher values of the efflux time (Figure 6). Using some non-dimensional coordinates

$t_{r e l}=\frac{t-t_{0}}{T}, \quad v_{r e l}=\frac{V(t)-V\left(t_{0}\right)}{V(T)}$,

the comparison of the observed curves can be done in a much easier way (Fig. 7). In (30) $t_{0}$ is the efflux time of the first $100 \mathrm{~cm}^{3}$ (that is $V\left(t_{0}\right)$ ) and $T$ is the efflux time of the standardized $200 \mathrm{~cm}^{3}$ (that is $V(T)$ ) of the studied liquid.

The slope of the non-dimensional curves is a kind of non-dimensional volumetric flow-rate, as the slope of the curves represented on Figure 6 is the true $Q_{V}(t)$ function.
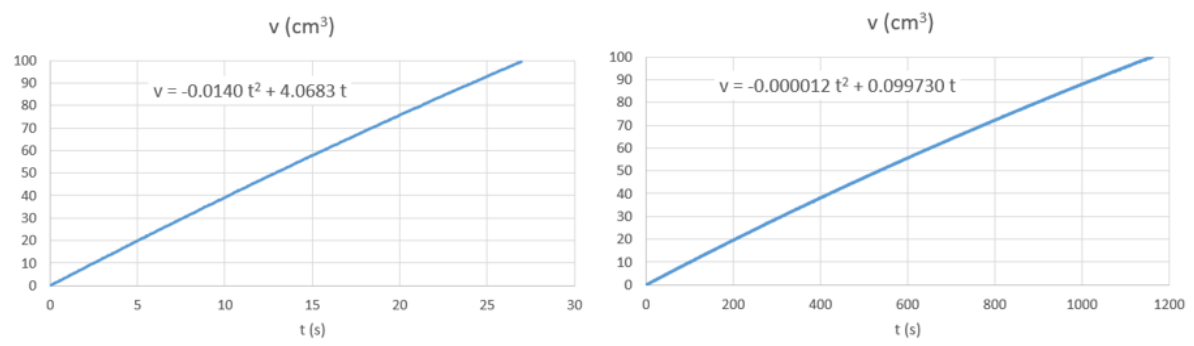

Figure 6

Outflow vs. efflux time of the last $100 \mathrm{~cm} 3$ for water and $10 \mathrm{~W} 40$ motor oil at $20^{\circ} \mathrm{C}$

Both the true and the non-dimensional volume-time curves (their shape), indicate that the flow rate and the efflux time of a certain volume, are given by some-nonlinear functions and there is no proportionality between these quantities and the viscosity of the liquid.
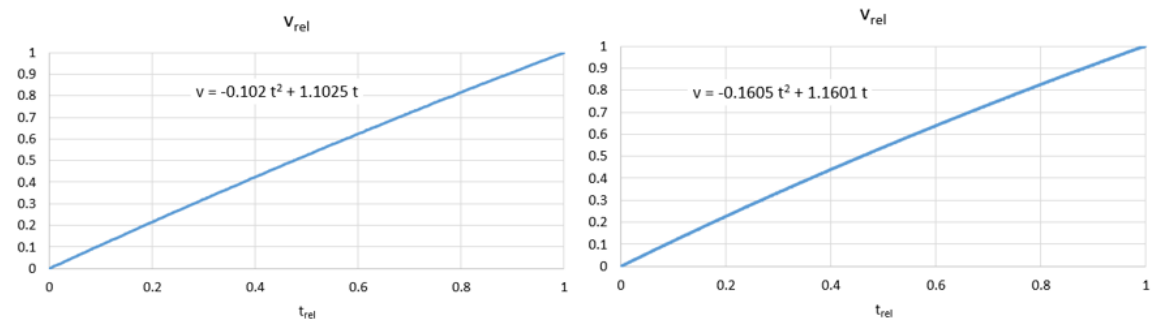

Figure 7

Outflow vs. efflux time of the last $100 \mathrm{~cm} 3$ for water and $10 \mathrm{~W} 40$ motor oil at $20^{\circ} \mathrm{C}$ in nondimensional coordinates 
As a final conclusion, the following can be stated:

- By implementing the mass measurement the efflux-type viscometers can be fully automatized without their structural modification. In this way the measuring errors can be reduced and the viscosity can be obtained with higher accuracy because the human factor (reading errors, reaction time) is completely eliminated. The "cooling error" and those related to the precision of the measured volume using the collecting flask are also eliminated.

- Computing the Engler-viscosity from the efflux-time of the last $100 \mathrm{~cm}^{3}$ of the liquids is a more consistent method because it reduces the effects of the unsteady flow, especially for less viscous liquids, such as, water.

- A properly accurate relation between the "true" kinematic viscosity and efflux time can be obtained only with a multipoint calibration using liquids with known viscosity. This can be obtained only with a multipoint calibration using liquids with known viscosity.

- Acquired data is the evolution in time of the outflow mass and volume. This makes possible a detailed analysis of the outflow. The physical meaning of the coefficients of the polynomial approximation of the observed $V(t)$ curves, will be investigated in future research work.

\section{References}

[1] Winslow H. Herschel: The Redwood viscometer, U.S. Government Printing Office, 1922 (republished by the Forgotten Books, 2019, ISBN 9781528511605)

[2] Winslow H. Herschel: Determination of Absolute Viscosity by Short-tube Viscosimeters in "Technologic Papers of the Bureau of Standards", U.S. Government Printing Office, 1917 (republished by the Forgotten Books, 2018, ISBN 978-1390929072)

[3] S. V. Gupta: Viscometry for Liquids: Calibration of Viscometers, Springer Series in Materials Science, Vol. 194, 2014, ISBN 978-3-319-04858-1, https://doi.org/10.1007/978-3-319-04858-1

[4] Viswanath, D. S., Ghosh, T., Prasad, D. H. L., Dutt, N. V. K., Rani, K. Y: Viscosity of Liquids. Theory, Estimation, Experiment, and Data, Springer, Dordrecht, 2007, ISBN 978-1-4020-5481-5, https://doi.org/10.1007/978-14020-5482-2

[5] L. Schiller: The Engler Viscometer and the Theory of Laminar Flow at the Entrance of a Tube, Journal of Rheology, 3(2), 1932, pp. 212-216, https://doi.org/10.1122/1.2116453

[6] Avia Semiconductor: Datasheet of the HX711 load cell amplifier https://cdn.sparkfun.com/datasheets/Sensors/ForceFlex/hx711_english.pdf 